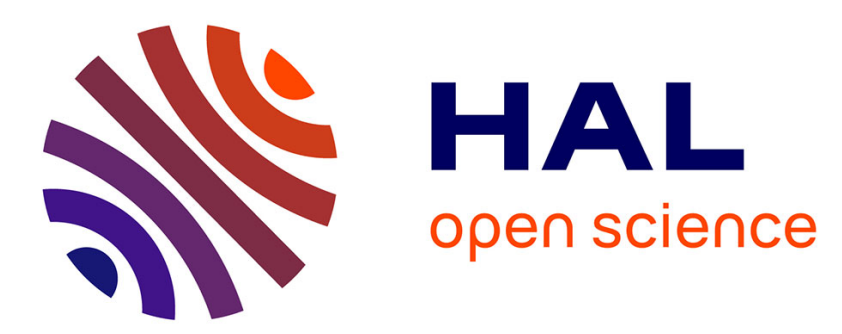

\title{
Hypermutability in clinical isolates of is uncommon and is unrelated to ciprofloxacin resistance
}

\author{
S. Aathithan, G.L. French
}

\section{To cite this version:}

S. Aathithan, G.L. French. Hypermutability in clinical isolates of is uncommon and is unrelated to ciprofloxacin resistance. International Journal of Antimicrobial Agents, 2010, 36 (3), pp.239. 10.1016/j.ijantimicag.2010.04.010 . hal-00608988

\section{HAL Id: hal-00608988 \\ https://hal.science/hal-00608988}

Submitted on 17 Jul 2011

HAL is a multi-disciplinary open access archive for the deposit and dissemination of scientific research documents, whether they are published or not. The documents may come from teaching and research institutions in France or abroad, or from public or private research centers.
L'archive ouverte pluridisciplinaire HAL, est destinée au dépôt et à la diffusion de documents scientifiques de niveau recherche, publiés ou non, émanant des établissements d'enseignement et de recherche français ou étrangers, des laboratoires publics ou privés. 


\section{Accepted Manuscript}

Title: Hypermutability in clinical isolates of Klebsiella pneumoniae is uncommon and is unrelated to ciprofloxacin resistance $^{1}$

Authors: S. Aathithan, G.L. French

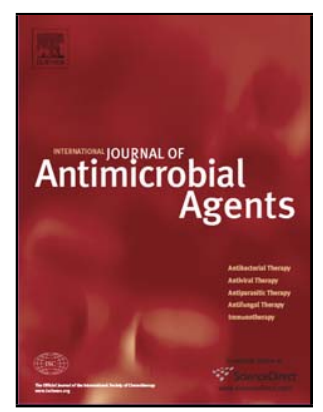

PII:

S0924-8579(10)00198-6

DOI: doi:10.1016/j.ijantimicag.2010.04.010

Reference: ANTAGE 3314

To appear in: International Journal of Antimicrobial Agents

Received date: $\quad 16-2-2010$

Revised date: 26-4-2010

Accepted date: $\quad$ 27-4-2010

Please cite this article as: Aathithan S, French GL, Hypermutability in clinical isolates of Klebsiella pneumoniae is uncommon and is unrelated to ciprofloxacin resistance ${ }^{1}$, International Journal of Antimicrobial Agents (2008), doi:10.1016/j.ijantimicag.2010.04.010

This is a PDF file of an unedited manuscript that has been accepted for publication. As a service to our customers we are providing this early version of the manuscript. The manuscript will undergo copyediting, typesetting, and review of the resulting proof before it is published in its final form. Please note that during the production process errors may be discovered which could affect the content, and all legal disclaimers that apply to the journal pertain. 


\title{
Hypermutability in clinical isolates of Klebsiella pneumoniae is uncommon and is unrelated to ciprofloxacin resistance
}

\author{
S. Aathithan *, G.L. French
}

King's College London, Department of Infectious Diseases, Division of Immunology, Infection and Inflammatory Diseases, School of Medicine, St Thomas' Hospital, London SE1 7EH, UK

ARTICLE INFO

Article history:

Received 16 February 2010

Accepted 27 April 2010

Keywords:

Enterobacteriaceae

Klebsiella

Random hypermutation

Fluoroquinolone resistance

* Corresponding author. Tel.: +44 207188 3127; fax: +44 2079280730.

E-mail address: sivasampu.aathithan@kcl.ac.uk (S. Aathithan).

Page $\mid 1$ 
Part of this study was presented at the 19th European Congress of Clinical Microbiology and Infectious Diseases (ECCMID), 16-19 May 2009, Helsinki, Finland (abstract P1481).

Page | 2 


\section{ABSTRACT}

We investigated hypermutability in Klebsiella pneumoniae and its association with ciprofloxacin resistance and mutations in the quinolone resistance-determining region (QRDR). Sixty-four strains of K. pneumoniae isolated in London, UK, between 1995 and 2002 with widely differing ciprofloxacin minimum inhibitory concentrations (MICs) and known gyrA and parC sequences were tested for mutation frequencies by selection with rifampicin. Only three hypermutable (frequency $\geq 10^{-6}$ ) strains were identified, with ciprofloxacin MICs of $0.25,8$ and $64 \mu \mathrm{g} / \mathrm{mL}$. There was no relationship between hypermutation and the ciprofloxacin MIC or QRDR mutations. Screening selected strains with streptomycin did not reveal any hypermutators, and screening with ciprofloxacin identified only two of the three hypermutators identified by rifampicin. Hypermutation in K. pneumoniae is uncommon and does not contribute to accumulation of QRDR mutations or directly to ciprofloxacin resistance.

Page | 3 


\section{Introduction}

Bacteria generally exhibit a mutation frequency of $10^{-10}$ per replicated base pair [1]. Strains that exhibit an elevated mutation frequency are said to be hypermutable $[2,3]$. There is no consensus on the mutation frequency above which an isolate is considered to be hypermutable [3], but a frequency of $>10^{-6}$ is widely accepted [4] and this value has been used in the present study.

The methyl-directed mismatch repair (MMR) system, which involves the genes mutS, mutL, mutH and mutU, normally corrects illegitimate DNA replication [5] and hypermutator phenotypes are thought to arise as a result of mutations in these genes. It is postulated that hypermutators develop antibacterial resistance at a higher frequency than MMR-proficient cells when exposed to antimicrobials $[4,6,7]$. This may be due to an increased frequency of target site mutations or to changes in other genes, such as those associated with efflux pump activity. Hypermutability may also bring about mutation in a single global gene such as mar, which can lead to the development of simultaneous multiple antibacterial resistance [8]. It has therefore been proposed that hypermutators have a higher rate of antimicrobial resistance and multiple resistance than normal clones and that hypermutation plays a role in the emergence of resistance $[2,4,6,7]$. This has been supported by several studies of different bacterial species and antimicrobial agents [4,7], but not by others [9]. Thus, the general role of hypermutability in the emergence of clinically relevant antibacterial resistance remains uncertain.

Page $\mid 4$ 
The incidence of fluoroquinolone resistance in Enterobacteriaceae has steadily increased since these agents were introduced into clinical practice $[10,11]$. The commonest mechanism involved, and the one associated with the highest levels of resistance, is mutations in the quinolone resistance-determining region (QRDR) of the topoisomerase genes, especially gyrA and parC. Accumulation of multiple mutations in this region is associated with increasing fluoroquinolone resistance [11]. We hypothesised that if hypermutability is associated with the development of fluoroquinolone resistance, then hypermutators should have more QRDR mutations and higher fluoroquinolone minimum inhibitory concentrations (MICs) than nonhypermutators.

We previously determined the QRDR mutations associated with ciprofloxacin resistance in a collection of distinct clinical isolates of Klebsiella pneumoniae with a range of ciprofloxacin MICs [12]. In this study, the prevalence of hypermutation in this isolate collection was investigated.

\section{Materials and methods}

2.1. Organisms, antimicrobials, culture media and antimicrobial susceptibility testing Sixty-four clinical isolates of K. pneumoniae isolated in London, UK, between 1995 and 2002, chosen to represent distinct isolates by biochemical and antibacterial susceptibility profiles and repetitive sequence-based polymerase chain reaction (repPCR) screening, were investigated [12]. Mutations in gyrA and parC were identified by sequencing [12]. Ciprofloxacin, rifampicin and streptomycin MICs were

Page | 5 
determined by agar dilution. MICs of chloramphenicol (CHL), tetracycline (TET) and trimethoprim (TMP) were determined for 26 isolates by Etest. Escherichia coli NCTC 10418 and K. pneumoniae NCTC 009633 were used as standards and as controls in the hypermutability studies.

\subsection{Hypermutability tests}

\subsubsection{Detection of hypermutability using rifampicin or streptomycin}

Hypermutability was investigated by a modified method of Oliver et al. [4]. Overnight, aerobic, $37^{\circ} \mathrm{C}$ shake cultures in Luria-Bertani (LB) broth were used to make cell suspensions of ca. $10^{10}$ colony-forming units (CFU)/mL. The inoculum for the broth culture was taken from an overnight colony on horse blood agar that was streaked to single colonies to check for colony homogeneity. For each isolate, $100 \mu \mathrm{L}$ of cell suspension in phosphate-buffered saline (PBS) were spread on two LB agar plates containing either $100 \mu \mathrm{g} / \mathrm{mL}$ rifampicin (ca. 3-6x the rifampicin MICs for the majority of the isolates) or $200 \mu \mathrm{g} / \mathrm{mL}$ streptomycin (two- to several-fold the streptomycin MICs). Plates were incubated at $37^{\circ} \mathrm{C}$ for $24-48 \mathrm{~h}$ and the average number of colonies appearing on the two plates was recorded. Total viable counts were determined on antibacterial-free plates. Each isolate was tested twice and the average mutation frequency was calculated. Putative hypermutable isolates were tested twice more to confirm that this was not a random event. Mutational stability was tested by plating 24 colonies of each isolate on agar containing $100 \mu \mathrm{g} / \mathrm{mL}$ rifampicin.

Page | 6 


\subsubsection{Detection of hypermutability using ciprofloxacin}

A further study was performed to determine hypermutability using ciprofloxacin selection. This study was performed with 12 selected clinical isolates comprising highly resistant, resistant and susceptible phenotypes, with multiple, single and no QRDR mutations, respectively, including the three isolates that were identified as hypermutable by the rifampicin study. Cell suspensions of overnight LB broth cultures of ca. $10^{10} \mathrm{CFU} / \mathrm{mL}$ were made in PBS and $100 \mu \mathrm{L}$ was spread on two IsoSensitest agar (ISA) plates containing ciprofloxacin at $4 \times$ the ciprofloxacin MIC and on plates without antimicrobial. Plates were incubated at $37^{\circ} \mathrm{C}$ and colonies were counted after 24, 48 and $72 \mathrm{~h}$. The frequency of ciprofloxacin-resistant mutant formation was averaged from three experiments. For each isolate, 12 randomly selected colonies from the ciprofloxacin selection plates were tested for stability by subculturing on ISA containing ciprofloxacin at $4 \times$ the ciprofloxacin MIC. Additionally, 12 randomly selected colonies of isolates 56 and 38 (hypermutable by the rifampicin study) were subcultured on plates containing $32 \mu \mathrm{g} / \mathrm{mL}$ ciprofloxacin to identify highlevel ciprofloxacin-resistant progeny.

\section{Results}

\subsection{Antimicrobial susceptibilities and hypermutability assays}

Ciprofloxacin MICs ranged from $0.016 \mu \mathrm{g} / \mathrm{mL}$ to $>512 \mu \mathrm{g} / \mathrm{mL}$. Rifampicin MICs ranged from $4 \mu \mathrm{g} / \mathrm{mL}$ to $>256 \mu \mathrm{g} / \mathrm{mL}$, with the majority (75\%) being either $16 \mu \mathrm{g} / \mathrm{mL}$ or $32 \mu \mathrm{g} / \mathrm{mL}$ (ca. $6 \times$ or $3 \times$ less than the $100 \mu \mathrm{g} / \mathrm{mL}$ hypermutability screening concentration). Streptomycin MICs ranged from $0.25 \mu \mathrm{g} / \mathrm{mL}$ to $>256 \mu \mathrm{g} / \mathrm{mL}$ and Page $\mid 7$ 
varied widely. However, 16 of the isolates had similar streptomycin susceptibility and these were used in the streptomycin hypermutability analysis.

3.2. Screening for hypermutable isolates using rifampicin and streptomycin, and relationship of hypermutability with ciprofloxacin minimum inhibitory concentration and quinolone resistance-determining region mutations

As shown in Fig. 1, isolates 2, 38 and 56 were hypermutable with respect to rifampicin. None of the 16 selected isolates tested (including 2 that were hypermutable with rifampicin) was hypermutable with respect to streptomycin. Fig. 2 shows that there was no association between ciprofloxacin MICs and rifampicin hypermutability; there was also no association between the number of mutations in the QRDR and rifampicin hypermutability (data not shown). Amongst the three isolates hypermutable with rifampicin, isolate 2 had a ciprofloxacin MIC of $64 \mu \mathrm{g} / \mathrm{mL}$ and three QRDR alterations (Ser83Tyr and Asp87Ala in GyrA and Ser80lle in ParC); isolate 56 had a ciprofloxacin MIC of $8 \mu \mathrm{g} / \mathrm{mL}$ with a single Asp87Gly alteration in GyrA; and isolate 38 had a ciprofloxacin MIC of $0.25 \mu \mathrm{g} / \mathrm{mL}$ with no QRDR mutations either in gyrA or parC [12].

Although some isolates $(34,101,19,72,46$ and 102) exhibited elevated mutation frequencies $\left(1.4 \times 10^{-7}-4.7 \times 10^{-7}\right)$ in the streptomycin assay, none was found to be hypermutable or had an elevated mutation frequency in the rifampicin assay, which is considered to be the 'gold standard'. With the ciprofloxacin assay, isolate 54 showed a near-hypermutant phenotype $\left(7.2 \times 10^{-7}\right)$, but all the hypermutant 
progenies were unstable and it was found to be a non-hypermutator by the other two assays.

3.3. Screening for hypermutable isolates using ciprofloxacin, and relationship of hypermutability with ciprofloxacin minimum inhibitory concentration and quinolone resistance-determining region mutations

The ciprofloxacin MICs of the 12 isolates tested for hypermutability by ciprofloxacin screening ranged from $0.032 \mu \mathrm{g} / \mathrm{mL}$ to $128 \mu \mathrm{g} / \mathrm{mL}$. They comprised three ciprofloxacin susceptibility groups: six isolates with multiple QRDR mutations that were highly resistant to ciprofloxacin; three isolates with single mutations and lowlevel resistance to ciprofloxacin; and three ciprofloxacin-susceptible isolates with no mutations. The frequency of ciprofloxacin-resistant mutants at $4 \times$ the ciprofloxacin MIC varied more than 1000 -fold amongst these isolates. Isolates 2 and 38 yielded resistant mutants at frequencies of $4.4 \times 10^{-6}$ and $6.3 \times 10^{-6}$, respectively, and were thus considered hypermutable. Isolate 56 , which had been identified as hypermutable in the rifampicin study, was not identified as hypermutable with ciprofloxacin. The six highly ciprofloxacin-resistant isolates (MICs $>16 \mu \mathrm{g} / \mathrm{mL}$ ) had multiple mutations in the QRDR, but only isolate 2 was hypermutable by ciprofloxacin screening. Two of the six highly resistant isolates (54 and 45), although not hypermutable, exhibited an elevated mutation frequency of ca. $10^{-7}$. The three ciprofloxacin-susceptible isolates with MICs of $<0.5 \mu \mathrm{g} / \mathrm{mL}$ had no mutations in the QRDR, but one (isolate 38) was hypermutable by ciprofloxacin screening and was also hypermutable by the rifampicin assay. Another ciprofloxacin-susceptible isolate (isolate 57) had an elevated mutation frequency of $3.8 \times 10^{-7}$; the ciprofloxacinPage $\mid 9$ 
susceptible K. pneumoniae reference strain, with no QRDR mutations, exhibited a similar elevated mutation frequency. In the third group of three isolates with ciprofloxacin MICs of $1-8 \mu \mathrm{g} / \mathrm{mL}$, each had one mutation in the QRDR; none was hypermutable or showed an elevated mutation frequency by ciprofloxacin screening. None of the 12 colonies of the ciprofloxacin-susceptible but hypermutable isolate 38 , randomly selected from the $1 \mu \mathrm{g} / \mathrm{mL}$ ciprofloxacin screening plate, grew on $32 \mu \mathrm{g} / \mathrm{mL}$ ciprofloxacin. This suggested that these mutant progenies did not inherit multiple QRDR mutations (see discussion), but this was not confirmed by sequencing.

\subsection{Detection of hypermutability using a modified Etest method}

When the Etest was used to determine the MICs of CHL, TET and TMP, a large number of normal-sized squatter colonies was observed within the inhibition zones around the CHL, TET and TMP strips with isolate 38, which was susceptible to these agents and had been identified as hypermutable by rifampicin and ciprofloxacin assays. We concluded that the 'squatter colonies' represented resistant mutant progeny and hypothesised that Etest strips might be used as a simple way to screen for hypermutability with a range of antimicrobials. We therefore tested the presumptive hypermutable isolates 38 and 56 (but not isolate 2, which was highly resistant to all the antibacterials used in the Etest) twice by Etest using ca. $10^{7}$ CFU/89-mm diameter plate (i.e. $10^{7} \mathrm{CFU} / 62 \mathrm{~cm}^{2}$ ).

As shown in Fig. 3, isolate 38 produced $>30$ squatter colonies within each inhibition zone, equivalent to ca. 30 colonies $/ 1.25 \times 10^{6} \mathrm{CFU}$ seeded, giving a mutation frequency of ca. $2.4 \times 10^{-5}$ with all three antibacterials. This phenomenon was not Page | 10 
seen with isolate 56 , another presumptive hypermutable isolate selected with rifampicin, or with other non-hypermutable isolates.

\section{Discussion}

A collection of 64 distinct $K$. pneumoniae isolates expressing a range of ciprofloxacin MICs and containing multiple, single or no mutations in the QRDR was investigated. Hypermutability (mutation frequency of $\geq 10^{-6}$ ) was uncommon and there was no association between hypermutability and ciprofloxacin resistance or QRDR mutations. Only $3(<5 \%)$ of the 64 isolates were identified as hypermutable by rifampicin screening. Amongst the 24 isolates with multiple QRDR mutations and high-level ciprofloxacin resistance, only one was hypermutable by the rifampicin assay. Furthermore, hypermutability was found more frequently (1 in 3$)$ in isolates with no mutations in the QRDR than in those with multiple mutations (1 in 6). We conclude that there was no causal association between hypermutability and multiple QRDR mutations or high-level ciprofloxacin resistance.

Ciprofloxacin was not used for the initial hypermutability screening because of the possibility of mutants arising as a result of ciprofloxacin SOS induction [13,14]. A relatively high proportion of tested isolates $(3 / 12 ; 25 \%)$ exhibited an elevated frequency of mutation (ca. $10^{-7}$ ) with ciprofloxacin, but this was much lower with rifampicin $(2 / 64 ; 3.1 \%)$. This high prevalence of elevated mutation frequency with ciprofloxacin may be due to the SOS-inducing effect of the drug [13,14].

Page $\mid 11$ 
Isolate 56 appeared hypermutable with rifampicin but not with ciprofloxacin or streptomycin, indicating that apparent hypermutability varies with the agent used for screening. Rifampicin, which generated the least number of isolates with an elevated mutation frequency, appeared to be the best agent for identifying hypermutation in these isolates.

The mutant colonies selected by rifampicin were always stable when subcultured on agar containing $100 \mu \mathrm{g} / \mathrm{mL}$ rifampicin. However, many of the apparently resistant colonies that formed on the $4 \times$ ciprofloxacin MIC screening plates were unstable when subcultured at the same ciprofloxacin concentration. This striking difference in stability suggests that some or all of the colonies on the $4 \times$ ciprofloxacin MIC plates were only transiently resistant or were only 'phenotypic mutators', possibly due to induction of SOS by ciprofloxacin. This indicates that ciprofloxacin is not a reliable agent for identifying hypermutators.

We had previously found that all the isolates in this collection with ciprofloxacin MICs of $\geq 16 \mu \mathrm{g} / \mathrm{mL}$ had multiple QRDR mutations [12]. We postulated that progeny of hypermutable isolates selected by ciprofloxacin and resistant to $32 \mu \mathrm{g} / \mathrm{mL}$ ciprofloxacin were likely to have multiple QRDR mutations. Colonies of the hypermutable isolate $38(\mathrm{MIC}=0.25 \mu \mathrm{g} / \mathrm{mL})$ that grew on the $1 \mu \mathrm{g} / \mathrm{mL}$ ciprofloxacin hypermutability screening plates were therefore tested for high-level resistance. None of the 12 randomly selected colonies grew on $32 \mu \mathrm{g} / \mathrm{mL}$ ciprofloxacin, suggesting that hypermutability did not give rise to progenies with multiple mutations in the QRDRs in this isolate.

Page $\mid 12$ 
We also tested for hypermutation selected by other antimicrobials using Etest strips for 26 of the 64 isolates. This strategy was adopted following the observation that the persistently hypermutant isolate 38 consistently yielded a large number of normalsized squatter colonies within the Etest zones of inhibition for CHL, TET and TMP (Fig. 3). Amongst the three hypermutable isolates identified by rifampicin screening, the Etest method showed that only isolate 38 consistently expressed the hypermutable phenotype with $\mathrm{CHL}$, TET and TMP. Thus, only isolate 38 was consistently hypermutable with a range of antimicrobials. We propose that hypermutability should be tested by selection with a range of antimicrobial classes and that this can be done conveniently by Etest. Thus, our study lends further support for the proposal that the Etest, which is simpler than the commonly used hypermutation assay, could be used to identify hypermutators in clinical laboratories [15]. Looking for hypermutation in routine Etest-based MIC plates could help alert clinicians about the possibility of enhanced resistance development in isolates that produce normal-sized resistant colonies in the zone of inhibition.

In conclusion, this study found that: (i) hypermutability was uncommon in clinical isolates of $K$. pneumoniae in London; (ii) hypermutability occurred randomly amongst ciprofloxacin-susceptible and -resistant strains and there was no evidence to support the hypothesis that hypermutability was associated with or contributed to the development of multiple mutations in the QRDR or resistance to ciprofloxacin; (iii) the progeny of hypermutable isolates did not show high-level ciprofloxacin resistance, suggesting that hypermutability did not produce multiple mutations in the QRDR; (iv) the apparent hypermutability of an isolate depends on the agent used in Page | 13 
screening; (v) the use of ciprofloxacin in hypermutability screening studies may result in an apparent elevated mutation frequency, possibly due to SOS response of nonheritable nature; and (vi) putative hypermutant progeny should always be tested for stability before concluding that the parent strain is a true hypermutator.

\section{Acknowledgment}

The authors would like to thank Dr K. Shannon for advice and help with computer analysis of the data.

\section{Funding}

SA and the study were funded by King's College London (UK).

\section{Competing interests}

None declared.

\section{Ethical approval}

Not required.

Page | 14 


\section{References}

[1] Bridges BA. Hypermutation in bacteria and other cellular systems. Philos Trans R Soc Lond B Biol Sci 2001;356:29-39.

[2] Chopra I, O'Neill AJ, Miller K. The role of mutators in the emergence of antibioticresistant bacteria. Drug Resist Updat 2003;6:137-45.

[3] Hall LMC, Henderson-Begg SK. Hypermutable bacteria isolated from humans-a critical analysis. Microbiology 2006;152:2505-14.

[4] Oliver A, Canton R, Campo P, Baquero F, Blazquez J. High frequency of hypermutable Pseudomonas aeruginosa in cystic fibrosis lung infection. Science 2000;288:1251-3.

[5] Modrich P. Methyl-directed DNA mismatch correction. J Biol Chem 1989;264:6597-600.

[6] LeClerc JE, Li B, Payne WL, Cebula TA. High mutation frequencies among Escherichia coli and Salmonella pathogens. Science 1996;274:1208-11.

[7] Blazquez J. Hypermutation as a factor contributing to the acquisition of antimicrobial resistance. Clin Infect Dis 2003;37:1201-9.

[8] Barbosa TM, Levy SB. Differential expression of over 60 chromosomal genes in Escherichia coli by constitutive expression of MarA. J Bacteriol 2000;182:346774.

[9] Gutierrez O, Juan C, Perez JL, Oliver A. Lack of association between hypermutation and antibiotic resistance development in Pseudomonas aeruginosa isolates from intensive care unit patients. Antimicrob Agents Chemother 2004;48:3573-5.

Page $\mid 15$ 
[10] Landman D, Bratu S, Kochar S, Panwar M, Trehan M, Doymaz M, et al. Evolution of antimicrobial resistance among Pseudomonas aeruginosa, Acinetobacter baumannii and Klebsiella pneumoniae in Brooklyn, NY. J Antimicrob Chemother 2007;60:78-82.

[11] Hooper DC. Mechanisms of quinolone resistance. In: Hooper DC, Rubinstein E, editors. Quinolone antimicrobial agents. Washington, DC: ASM Press; 2003. p. $41-67$.

[12] Aathithan S. Mechanisms and genetics of ciprofloxacin resistance in Klebsiella pneumoniae [PhD thesis]. London, UK: University of London; 2008.

[13] Phillips I, Culebras E, Moreno F, Baquero F. Induction of the SOS response by new 4-quinolones. J Antimicrob Chemother 1987;20:631-8.

[14] Luo N, Sahin O, Lin J, Michel LO, Zhang Q. In vivo selection of Campylobacter isolates with high levels of fluoroquinolone resistance associated with gyrA mutations and the function of the CmeABC efflux pump. Antimicrob Agents Chemother 2003;47:390-4.

[15] Maciá MD, Borrell N, Perez JL, Oliver A. Detection and susceptibility testing of hypermutable Pseudomonas aeruginosa strains with the Etest and disk diffusion. Antimicrob Agents Chemother 2004;48:2665-72.

Page | 16 
Fig.1. Frequency of mutation with rifampicin in 64 Klebsiella pneumoniae isolates. Isolates are plotted on the $x$-axis and mutation frequencies are on the $y$-axis. Mutator strains are defined as having a mutation frequency of $\geq 10^{-6}$. Note that the isolate number on the $x$-axis does not correspond to the true isolate numbers, referred to in the text.

Fig. 2. Ciprofloxacin (CIP) minimum inhibitory concentrations (MICs) and frequency of generation of rifampicin-resistant mutants. $\square$, isolates with rifampicin MICs of $\leq 16$ $\mu \mathrm{g} / \mathrm{mL} ; \bullet$ isolates with a rifampicin MIC of $32 \mu \mathrm{g} / \mathrm{mL} ; \boldsymbol{\Lambda}$, isolates with rifampicin MICs of $\leq 64 \mu \mathrm{g} / \mathrm{mL}$.

Fig. 3. Hypermutable isolate 38 showing hypermutability using Etest strips. Strips from left to right: chloramphenicol; tetracycline; and trimethoprim. 
Isolate number

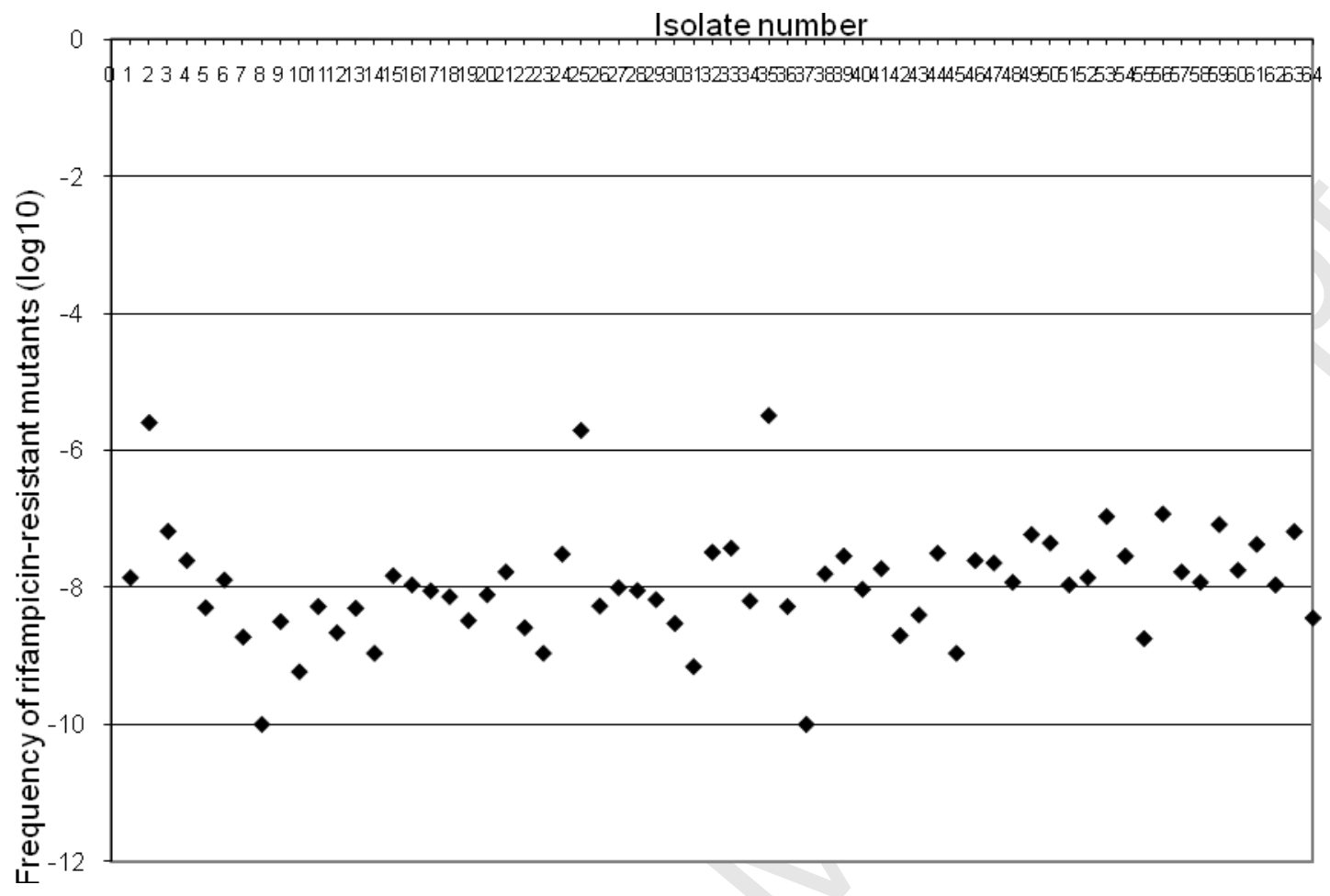

Page | 1 


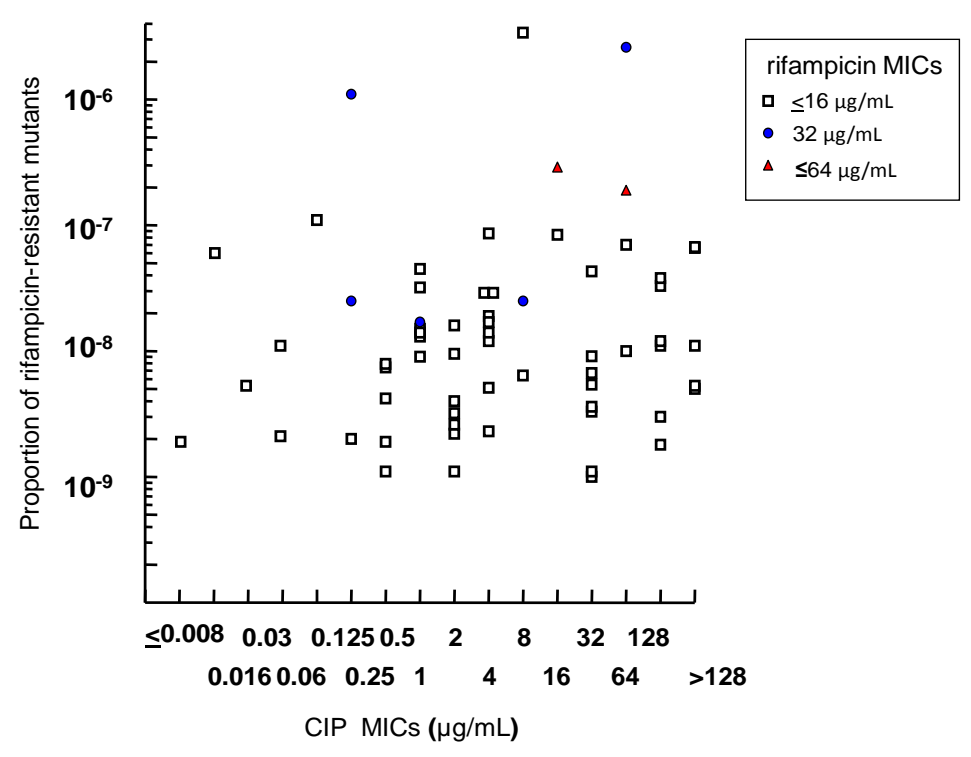




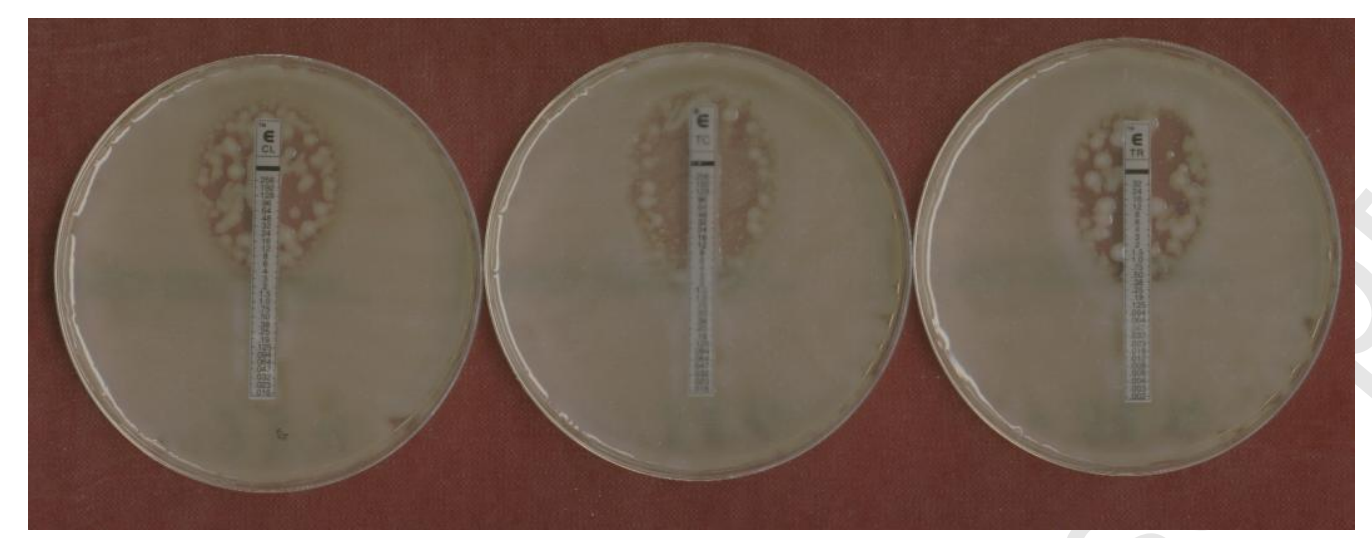

